\title{
Coherent Multi-Channel Ranging for Narrowband LPWAN: Simulation and Experimentation Results
}

\author{
Florian Wolf*\|, Jean-Baptiste Doré*, Xavier Popon*, \\ Sébastien de Rivaz*, François Dehmas* and Jean-Pierre Cances" \\ * CEA-Leti Minatec Campus, 17 rue des Martyrs, 38054 Grenoble Cedex 09, France \\ Email: \{florian.wolf, jean-baptiste.dore, xavier.popon, sebastien.derivaz, francois.dehmas\}@cea.fr \\ \| Université de Limoges, CNRS, XLIM, UMR 7252, 87000 Limoges, France \\ Email: \{florian.wolf, jean-pierre.cances\}@xlim.fr
}

\begin{abstract}
Precise radio based positioning for low power wide area networks remains a challenging research area due to narrowband signals and multipath propagation. Multi-channel ranging provides improved temporal resolution by coherent processing. While this technique has been applied to short range radio standards, no experimental demonstration for long range radio devices exists. The present paper introduces a hardware testbed for phase coherent multi-channel processing of narrowband signals. Simulations and preliminary experimental multi-channel results show a higher ranging precision (factor 20) over time based ranging. In a frequency flat channel with a $10 \mathrm{kHz}$ signal, precisions down to $10 \mathrm{~m}$ (phase based) and $200 \mathrm{~m}$ (time based) have been achieved. Precision degrades in multipath propagation scenarios.
\end{abstract}

Index Terms-Experimental testbed, LPWAN localization, range estimation, phase-of-flight, frequency hopping

\section{INTRODUCTION}

Precise localization in wireless sensor networks (WSN) has become an interesting research area in recent years. Adding position information to the wireless transmitted data will enable new applications [1] and hence it is included in current standardization studies [2]. Positioning based on the communication radio signals should neither increase energy consumption nor hardware complexity of the node, which are critical to low power wide area network (LPWAN) use cases.

LPWAN radio technologies apply narrowband modulation schemes (e.g. NB-IoT, LoRa, Sigfox), offering high receiver sensitivity, required for long range communication over several kilometers. However, localization is challenging due to low temporal resolution and the difficulty to resolve multipath.

Techniques to improve localization accuracy on the system level comprise network densification [3] to enrich the set of measurements and sophisticated algorithms (e.g. finger printing [4], machine learning and deep neural networks). Improving the single link ranging precision, can provide more accurate raw estimates for positioning on the system level.

Coherent multi-channel ranging [5] allows aggregating multiple sequentially transmitted narrowband signals to a virtual increased bandwidth, making it compatible with LPWAN radio transceivers. Theoretical studies [6], [7] have investigated asymptotic precision limits while implementations [8]-[12] have demonstrated feasibility and an improved accuracy over single channel time based ranging techniques. Table I sum-
TABLE I

Related EXPERIMENTAL WORK: PARAMETERS \& RANGING PRECISION

\begin{tabular}{|c|c|c|c|c|c|c|}
\hline $\begin{array}{l}\text { Related } \\
\text { work }\end{array}$ & in $\stackrel{f_{\mathrm{R}}}{\mathbf{G H z}}$ & $\begin{array}{c}R_{\text {comm }} \\
\text { in } \mathbf{~ m}\end{array}$ & $\begin{array}{l}B W_{\mathrm{sym}} \\
\text { in } \mathbf{M H z}\end{array}$ & $\begin{array}{l}B W_{\text {virt }} \\
\text { in } \mathbf{M H z}\end{array}$ & $\begin{array}{l}\sigma_{\mathrm{ToF}} \\
\text { in } \mathbf{m}\end{array}$ & $\begin{array}{l}\sigma_{\mathrm{PoF}} \\
\text { in m }\end{array}$ \\
\hline LoRa & 0.868 & 4000 & 0.125 & $\mathrm{n} / \mathrm{a}$ & 205 & $\mathrm{n} / \mathrm{a}$ \\
\hline Sigfox [4] & 0.868 & 3000 & 0.001 & $\mathrm{n} / \mathrm{a}$ & 1000 & $\mathrm{n} / \mathrm{a}$ \\
\hline WiFi [8] & $2.4,5$ & 15 & 40 & 2600 & 15 & 0.5 \\
\hline Zigbee [9], [12] & 2.4 & 9 & 2 & 80 & 2 & 0.2 \\
\hline$R F I D{ }_{[10]}$ & 0.900 & 2.4 & ? & 26 & - & 0.14 \\
\hline ATMEL [11] & 2.4 & 50 & $?$ & ? & - & 1.63 \\
\hline
\end{tabular}

marizes related experimental work, frequency $f_{\mathrm{R}}$, communication range $R_{\text {comm }}$, instantaneous signal bandwidth $B W_{\text {sym }}$, corresponding ranging precision $\sigma_{\mathrm{ToF}}$, virtual coherent multichannel bandwidth $B W_{\text {virt }}$ and its ranging precision $\sigma_{\mathrm{PoF}}$.

While [8] uses the Channel State Information (CSI) of commodity WiFi cards to extract relevant phase information, inphase/quadrature (IQ) data is processed on the FPGA in [9], [12] and stored for post processing in [10]. This latter approach, feasible for short packet transmissions, generates large amounts of data with narrowband LPWAN signals.

The present work aims to transpose the concept of coherent multi-channel ranging to LPWAN systems with signal bandwidths of $B W_{\mathrm{sym}}=10 \mathrm{kHz}$.

The contributions of this paper, to investigate the feasibility of coherent multi-channel ranging experimentally, are:

- A transceiver testbed, comprising a radio front-end and a FPGA performing digital up- and down-intermediate frequency mixing to reduce sampling rate while maintaining phase coherence over the virtual multi-channel bandwidth and IQ sample recording for post processing.

- Software simulations on top of the testbed to develop and benchmark ranging algorithms.

- Preliminary experimental results demonstrating coherent multi-channel ranging performances in a cabled frequency flat AWGN channel as function of noise.

This paper is organized as follows. Measurement principles and a basic ranging algorithm are outlined in Section II. The developed testbed architecture is described in Section III. Section IV gives simulation and experimental results, while Section V concludes with a perspective on further work. 


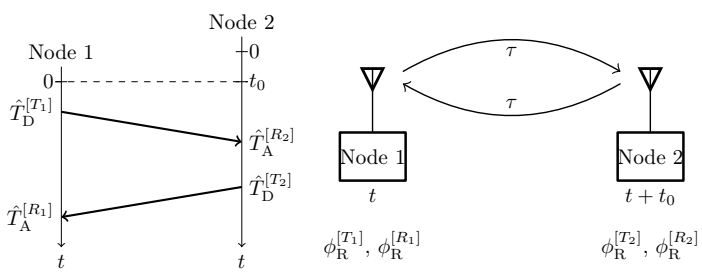

Fig. 1. Two-way time-of-flight (ToF) and phase-of-flight (PoF) measurement.

\section{Coherent Multi-Channel Ranging}

\section{A. Measurement principle}

The round trip time-of-flight (ToF) ranging measurement, as depicted in Fig. 1 can be expressed as follows

$$
\begin{aligned}
\mathrm{ToF} & =\left(\hat{T}_{\mathrm{A}}^{\left[R_{1}\right]}-\hat{T}_{\mathrm{D}}^{\left[T_{1}\right]}\right)-\left(\hat{T}_{\mathrm{D}}^{\left[T_{2}\right]}-\hat{T}_{\mathrm{A}}^{\left[R_{2}\right]}\right) \\
& =\left(\hat{T}_{\mathrm{D}}^{\left[T_{2}\right]}-t_{0}+\tau\right)+\left(\hat{T}_{\mathrm{D}}^{\left[T_{1}\right]}+t_{0}+\tau\right)-\left(\hat{T}_{\mathrm{D}}^{\left[T_{2}\right]}+\hat{T}_{\mathrm{D}}^{\left[T_{1}\right]}\right) \\
& =2 \tau .
\end{aligned}
$$

A two-way packet exchange [13] is required in order to eliminate unknown relative time reference $t_{0}$ on time of arrival (ToA) measurements $\hat{T}_{\mathrm{A}}^{[X]}$. Transmission times $\hat{T}_{\mathrm{D}}^{[X]}$ are known. Combining phase measurements $\hat{\phi}_{c}^{[X]}$ results in the phase-of-flight estimate $\left(\mathrm{PoF}_{c}\right)$ at channel frequency $c \Delta f+f_{\mathrm{R}}$

$$
\begin{aligned}
\mathrm{PoF}_{c}= & \hat{\phi}_{c}^{\left[R_{2}\right]}+\hat{\phi}_{c}^{\left[R_{1}\right]} \\
= & \left(-2 \pi c \Delta f t_{0}-2 \pi\left(c \Delta f+f_{\mathrm{R}}\right) \tau+\phi_{\mathrm{R}}^{\left[T_{1}\right]}-\phi_{\mathrm{R}}^{\left[R_{2}\right]}\right) \\
& \quad+\left(2 \pi c \Delta f t_{0}-2 \pi\left(c \Delta f+f_{\mathrm{R}}\right) \tau+\phi_{\mathrm{R}}^{\left[T_{2}\right]}-\phi_{\mathrm{R}}^{\left[R_{1}\right]}\right) \\
= & -2 \cdot 2 \pi\left(c \Delta f+f_{\mathrm{R}}\right) \tau+\Delta \phi_{\mathrm{R}} .
\end{aligned}
$$

with carrier frequency $f_{\mathrm{R}}$. A full description including carrier frequency offset (CFO) is given in [7]. CFO influence on ToF and PoF is neglected in the following. A multi-channel ranging packet exchange in the time-frequency domain is depicted in Fig. 2. A narrowband two-way exchange with an instantaneous waveform bandwidth $B W_{\text {sym }}$ is performed on $C$ channels sequentially, equivalent to sampling the channel transfer function $H(f)$. With channel intermediate frequency spacing $\Delta f$, a total virtual coherent multi-channel bandwidth $B W_{\text {virt }}=(C-1) \Delta f$ is covered. Range information can be extracted from the slope of the set $\mathrm{PoF}_{c}$ with $c \in[1 \ldots C]$

$$
d_{\mathrm{PoF}}=\tau c_{0}=-\frac{c_{0}}{4 \pi \Delta f} \cdot \frac{\Delta \mathrm{PoF}_{c}}{\Delta c}
$$

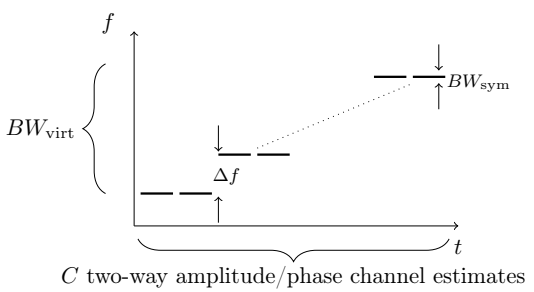

Fig. 2. Multi-channel ranging protocol aggregating $C$ sequentially transmitted narrowband signals $\left(B W_{\mathrm{sym}}\right)$ to a virtual bandwidth $B W_{\text {virt }}=(C-1) \Delta f$. with speed of light $c_{0}$. The channel transfer function ${ }^{1}$ can be reconstructed by

$$
\left.H(f)\right|_{\left(f=c \Delta f+f_{\mathrm{R}}\right)}=\hat{A}_{c} e^{j \mathrm{PoF}_{c}}
$$

with amplitude estimates $\hat{A}_{c}=\hat{A}_{c}^{\left[R_{1}\right]} \cdot \hat{A}_{c}^{\left[R_{2}\right]}$. Hence, in multipath scenarios it is preferred to convert $H(f)$ to the channel impulse response

$$
h(t)=\int_{-\infty}^{\infty} H(f) e^{-j 2 \pi f t} d f
$$

with the discrete inverse Fourier transform (iFFT). In order to detect the first path, a simple detection scheme identifies the first local maximum above a certain threshold $\gamma$ relative to and in a certain range $R_{\text {first }}$ before the global maximum in the channel impulse response $h(t)$.

Applying such a processing scheme, the following issues need to be considered [5]:

- Range resolution: $\Delta R=c_{0} /(2(C-1) \Delta f)=$ $c_{0} /\left(2 B W_{\text {virt }}\right)$. Maximize bandwidth $B W_{\text {virt }}$ for high range resolution and to distinguish close multipath.

- Range ambiguity, temporal aliasing: The phase based range measurement is ambiguous as the channel transfer function $H(f)$ is sampled with frequency spacing $\Delta f$. Therefore the reconstructed channel impulse response $h(t)$ becomes periodic with $R_{\max }=c_{0} /(2 \Delta f)$. Adapted system parameter design allows resolving ambiguities with two-way time based ranging measurements [14]. Furthermore, multipath components could potentially appear before the first path. Therefore the maximum unambiguous range $R_{\max }$ should be greater than the largest significant multipath component.

\section{$B$. Ranging metric extraction algorithm}

A BPSK signal $s_{0}$ of bit rate $R_{\mathrm{b}}=10 \mathrm{kbit} / \mathrm{s} \equiv B W_{\mathrm{sym}}=$ $10 \mathrm{kHz}$ has been assumed in this study. A Gold code preamble of length 32 assures good correlation for synchronization.

On each received signal $r_{c}^{[X]}$, as described in Section III, the algorithm depicted in Fig. 3 is applied. CFO $\hat{k}_{c}^{[X]}$ is estimated on the preamble sequence using an algorithm from [15]. Required time synchronization is achieved by differential detection. The CFO corrected signal is correlated with the transmit BPSK waveform $s_{0}$. Time of arrival $\hat{T}_{\mathrm{A}, c}^{[X]}$, amplitude $\hat{A}_{c}^{[X]}$ and phase $\hat{\phi}_{c}^{[X]}$ are extracted from the correlation peak.

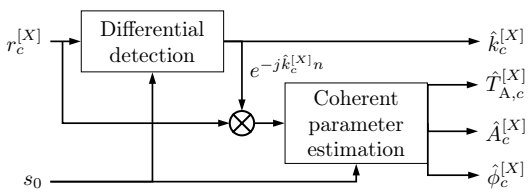

Fig. 3. Ranging metric extraction algorithm.

After processing over $C$ channels, the set $\left\{\hat{k}_{c}^{[X]}, \hat{T}_{\mathrm{A}, c}^{[X]}, \hat{A}_{c}^{[X]}, \hat{\phi}_{c}^{[X]}\right\}$ with $X \in\left[R_{1}, R_{2}\right]$ is available for further post processing and range extraction.

${ }^{1}$ Due to two-way ranging the square of the channel transfer function $H^{2}(f)$ is measured [12]. 


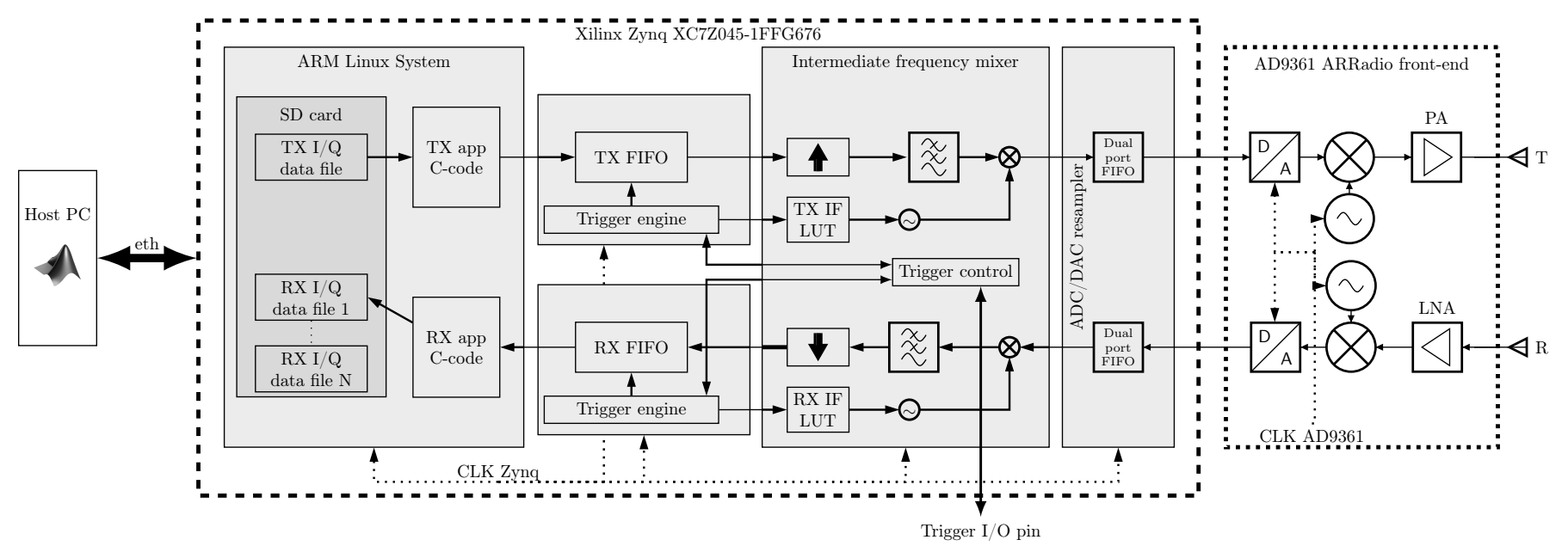

Fig. 4. SDR based coherent multi-channel ranging testbed architecture.

\section{TRANSCEIVER DESIGN}

The implementation of a coherent multi-channel ranging testbed is based on a software defined radio (SDR) platform comprising an Analog Devices AD9361 $2 \times 2$ TRX radio front end and a Zynq-045 Xilinx System on chip FPGA which integrates a dual Cortex-A9 ARM processor.

Fig. 4 shows the testbed architecture with the digital up-/ down-mixing stages implemented into the FPGA allowing to coherently process a $10 \mathrm{MHz}$ bandwidth by sequentially selecting $1 \mathrm{MHz}$ channels. The processor is dedicated to configuration purposes (RF/digital) as well as to transmit/receive IQ samples. The samples are in a second time processed by a dedicated software.

In the following the different parts of the testbed and their settings are explained in detail.

\section{A. AD9361 ARRadio front end configuration}

The radio front end is configured to maintain transmitter (TX) to receiver (RX) oscillator phase coherence by continuous operation of TX and RX phase lock loops (PLL) during the $C$ two-way packet exchanges. TX and RX local oscillator frequency is set to $f_{\mathrm{R}}=868 \mathrm{MHz}$, resulting in identical carrier frequency as both PLLs are driven from the same external clock $^{2}$. Initial phases $\phi_{\mathrm{R}}^{[X]}$ are arbitrary but constant. The automatic gain control (AGC) in the receiver is set to manual operation with a fixed gain to avoid delay and phase changes during the IQ data acquisition.

The 12 bit analog-to-digital converter (ADC) and the 12 bit digital-to-analog converter (DAC) are set to sampling rate $f_{\mathrm{s}, \mathrm{IF}}=20 \mathrm{MHz}$.

\section{B. FPGA design - Digital up-/down-mixing stages}

A first resampling stage acts as interface between the DAC/ADC and the digital up-/down-mixing blocks and adapts sampling frequency between the DAC/ADC and the FPGA processing.

\footnotetext{
${ }^{2}$ VCTCXO $40 \mathrm{MHz}$ (TXEAACSANF) with frequency accuracy of $2 \mathrm{ppm}$.
}

The following blocks are performing a factor 20 up-/downsampling and an intermediate frequency (IF) mixing. Intermediate frequency mixing is performed with a cordic that mixes $N_{\mathrm{S}}$ IQ samples to frequency $c \Delta f$ before changing to the next channel frequency in the look-up-table (LUT). Hence phase coherence between channel frequency switching is maintained. Synchronizing the TX/RX FIFOs with frequency selection enables coherent processing.

TX and RX IQ streams $\left(f_{\mathrm{s}, \mathrm{BB}}=1 \mathrm{MHz}\right)$ are buffered by large FIFOs between the FPGA and the ARM. The FIFOs can be triggered by 1) software (ARM controlled), 2) external GPIO pin or 3) onboard button. Perfect TX/RX intra-testbed synchronization is guaranteed by using the same trigger source. 1), 2) enable coarse inter-testbed time synchronization to limit acquisition time and to avoid the need to perform online synchronization between nodes on the radio signals.

The ARM processor runs TX and RX applications to manage IQ sample streams between the SD card and the FIFOs. The applications can be configured in master or slave mode to either initiate periodic two-way exchanges or to wait for the master via the trigger source.

\section{Received signal model}

The signals theoretically obtained in the RX FIFO of this transceiver design ${ }^{3}$ according to [7] are described by

$$
\begin{aligned}
r_{c}^{[X]}[n]= & A_{c}^{[X]} s_{0}\left(\frac{n}{f_{\mathrm{s}, \mathrm{BB}}}-T_{\mathrm{A}, c}^{[X]}\right) e^{j\left(k_{c}^{[X]} n+\phi_{c}^{[X]}\right)} \\
& +n_{c}^{[X]}[n]
\end{aligned}
$$

with with $n \in\left[0 \ldots N_{\mathrm{S}}-1\right]$ and noise $n_{c}^{[X]}$.

\section{APPLICATION}

\section{A. Simulation setup and results}

1) Setup: MATLAB simulations are conducted to develop and benchmark the ranging algorithms (see Section II) con-

\footnotetext{
${ }^{3}$ Neglecting jitter, ADC/DAC and IF mixer imperfections.
} 


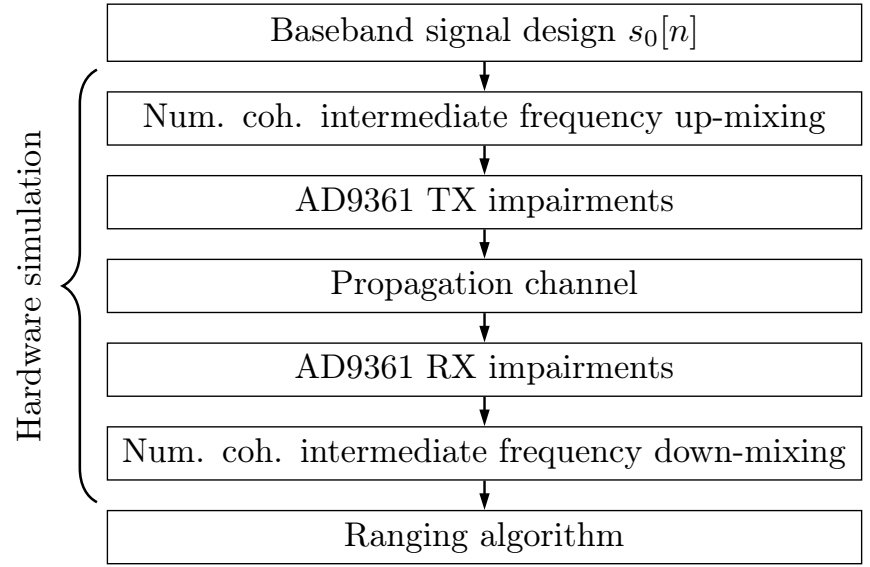

Fig. 5. Ranging algorithm benchmarking setup with simulated testbed signals.

sidering impairments introduced by digital intermediate frequency mixing (quantization, finite phase accuracy in cordic), radio front-end impairments and the influence of the propagation channel as depicted in Fig. 5. Digital coherent intermediate frequency up-/down-mixing blocks produce bitidentical outputs of the FPGA design. The AD9361 TX/RX blocks simulate unknown initial phase $\phi_{\mathrm{R}, c}^{[X]}$, unknown relative time reference $t_{0}$ and CFO $k_{c}^{[X]}$. Time-of-flight delay $\tau$ and multipath are included in the propagation channel block.

In the following, single channel time-of-flight $(\mathrm{ToF})$ based ranging (1) is compared to multi-channel (MC) time-of-flight $\left(\mathrm{ToF}_{\mathrm{MC}}\right)$ and the phase coherent multi-channel (PoF) (5) techniques. Simulations are conducted for a frequency flat channel and the ETSI typical urban (Tux) channel model [16] with root-mean-square (RMS) delay spread $\tau_{\text {rms }}=0.5 \mu$ s $\equiv$ $150 \mathrm{~m}$. Ranges are simulated between $0 \mathrm{~m}$ and $4 \mathrm{~km}$. A channel spacing of $\Delta f=200 \mathrm{kHz}$ and a total number of $C=16$ channels give $B W_{\text {virt }}=3.0 \mathrm{MHz}$ and result in a maximum unambiguous range $R_{\max }=750 \mathrm{~m}$ with a range resolution of $\Delta R=50 \mathrm{~m}$. This parameter choice derives from an empirical trade-off between multipath and range ambiguity resolvability as well as keeping system complexity reasonable (required packet transmissions $2 C$ ). Simulation results are averaged over $10^{3}$ independent realizations.

2) Frequency flat channel: Fig. 6 shows RMS ranging error over preamble symbol (Gold sequence of length 32) energy to noise spectral density $\left(E_{\mathrm{s}} / N_{0}\right)$ and equivalent BPSK $E_{\mathrm{b}} / N_{0}$ in a frequency flat channel for a signal bandwidth $B W_{\mathrm{sym}}=10 \mathrm{kHz}$. For both, time and phase based, ranging techniques at low $E_{\mathrm{s}} / N_{0} \mathrm{CFO}$ estimation does not provide sufficient accuracy for correct ranging parameter estimation. Single channel time-of-flight (ToF) ranging and averaging over the $C$ available time-of-flight measurements ( $\mathrm{ToF}_{\mathrm{MC}, \text { mean }}$ ) levels out for high $E_{\mathrm{s}} / N_{0}$ due to the limited sampling frequency $f_{\mathrm{s}, \mathrm{BB}}=1 \mathrm{MHz}$ giving an uniform distributed sampling resolution error [13]

$$
\sigma_{\mathrm{ToF}, \mathrm{f}_{\mathrm{s}, \mathrm{BB}}}=\frac{\sigma_{\mathrm{ToA}, \mathrm{f}_{\mathrm{s}, \mathrm{BB}}}}{\sqrt{2}}=\frac{c_{0}}{\sqrt{2} \sqrt{12} f_{\mathrm{s}, \mathrm{BB}}} \approx 61 \mathrm{~m} .
$$

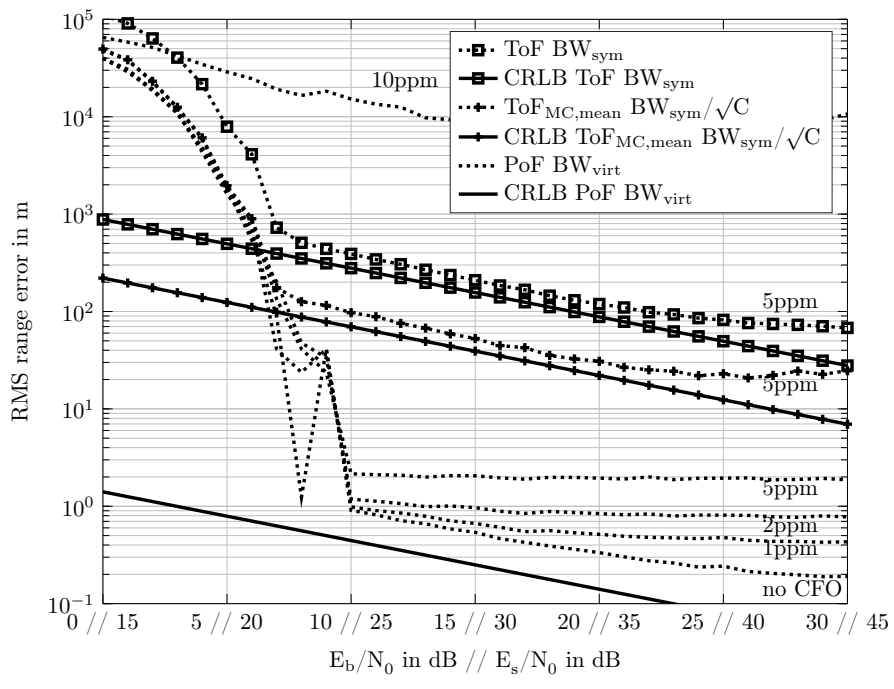

Fig. 6. Simulated ranging error in a frequency flat channel with a signal bandwidth $B W_{\text {sym }}=10 \mathrm{kHz}, C=16$ channels, a virtual multi-channel bandwidth $B W_{\text {virt }}=3.0 \mathrm{MHz}$ and a sampling frequency $f_{\mathrm{s}, \mathrm{BB}}=1 \mathrm{MHz}$.

Phase coherent processing (PoF) improves by one order of magnitude compared to time based approaches at equal energy. For no CFO estimation and high $E_{\mathrm{s}} / N_{0}$, a level out at $0.2 \mathrm{~m}$ due to the channel impulse response $h(t)$ range binning is observed. This can be controlled by appropriate IFFT zero padding in (5). Improvements are expected if CFO errors are compensated in future algorithms. Straight lines represent corresponding Cramer Rao lower bounds (CRLB) [6], [7].

3) Multipath channel: Simulation results for the ETSI typical urban (Tux) multipath scenario with a $B W_{\text {sym }}=$ $10 \mathrm{kHz}$ signal are depicted in Fig. 7. For first path detection (Section II-A), empirically determined threshold $\gamma=-7 \mathrm{~dB}$ and range $R_{\text {first }}=300 \mathrm{~m}$ showed best performances. These

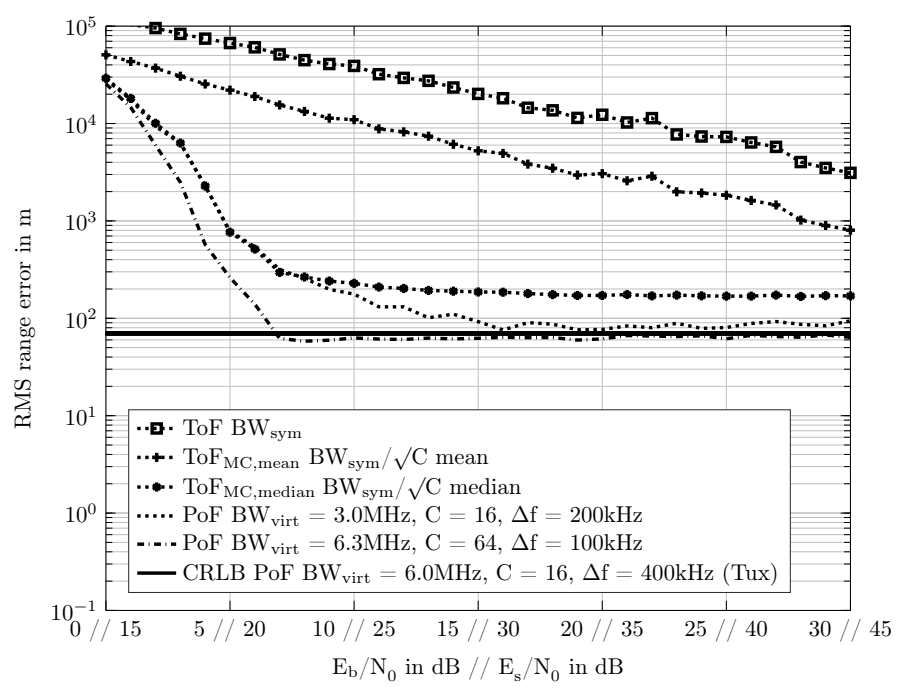

Fig. 7. Simulated ranging error in the ETSI typical urban (Tux) channel with a signal bandwidth $B W_{\mathrm{sym}}=10 \mathrm{kHz}, 1 \mathrm{ppm} \mathrm{CFO}$ and a sampling frequency $f_{\mathrm{s}, \mathrm{BB}}=1 \mathrm{MHz}$. 
values are in coherence with the Tux channel model characteristics delay spread and path gain ratio. Globally, ranging precision degrades compared to the frequency flat channel.

A closer study of the set of $C$ time-of-flight measurements reveals that range is over-estimated due to multipath and hence resulting in a positive biased ranging error. For a frequency flat channel no bias is observed. Therefore, the median $\left(\mathrm{ToF}_{\mathrm{MC}, \text { median }}\right)$ over the $C$ time-of-flight estimates drastically improves the ranging accuracy in multipath scenarios compared to averaging ( $\left.\mathrm{ToF}_{\mathrm{MC} \text {,mean }}\right)$. Coherent multichannel performances, with range ambiguity resolved by the median time-of-flight range, show a small improvement compared to the time based approach. Increasing the number of utilized channels $C=64$ with a frequency spacing $\Delta f=100 \mathrm{kHz}$, further improves coherent multi-channel performances. Whereas, the median on time-of-flight estimates $\left(\mathrm{ToF}_{\mathrm{MC} \text {,median }}\right)$ does not improve with more channels $C$. Coherent multi-channel precision approximately reaches the multipath CRLB [7].

\section{B. Experimental setup and results}

1) Setup: The experimental setup for two-way phase coherent multi-channel ranging is depicted in Fig. 8. A variable attenuator with attenuation $A_{\mathrm{V}, \mathrm{dB}}$ allows to control received power $P_{\mathrm{RX}, \mathrm{dBm}}$. The TX power is fixed to $P_{\mathrm{TX}, \mathrm{dBm}}=-71.5 \mathrm{dBm}$. The receiver low noise amplifier (LNA) is set to maximum gain, resulting in a minimum noise figure $N F_{\mathrm{dB}}=2.5 \mathrm{~dB}$ according to the datasheet. $E_{\mathrm{b}} / N_{0}$ and attenuation $A_{\mathrm{V}, \mathrm{dB}}$ are linked as follows

$$
\begin{aligned}
\frac{E_{\mathrm{b}}}{N_{0}}= & P_{\mathrm{TX}, \mathrm{dBm}}-A_{\mathrm{V}, \mathrm{dB}} \\
& \quad-(k T)_{\mathrm{dBm} / \mathrm{Hz}}-R_{\mathrm{b}, \mathrm{dBHz}}-N F_{\mathrm{dB}} \\
= & 60 \mathrm{~dB}-A_{\mathrm{V}, \mathrm{dB}},
\end{aligned}
$$

with Boltzmann constant $k$ and sytem temperature $T$, $(k T)_{\mathrm{dBm} / \mathrm{Hz}} \approx-174 \mathrm{dBm} / \mathrm{Hz}$. Experimental results are averaged over $10^{2}$ acquisitions.

2) Delay and phase calibration: In order to account for unknown digital filter- and analog delays in the AD9361 TRX as well as to adjust for unknown radio front-end transfer functions, an one-time (factory) calibration measurement is

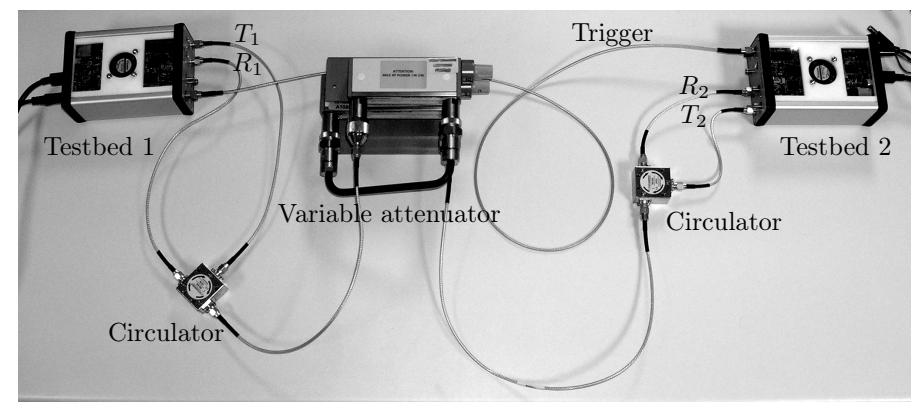

Fig. 8. Experimental setup for coherent multi-channel two-way ranging in a cabled frequency flat AWGN channel.

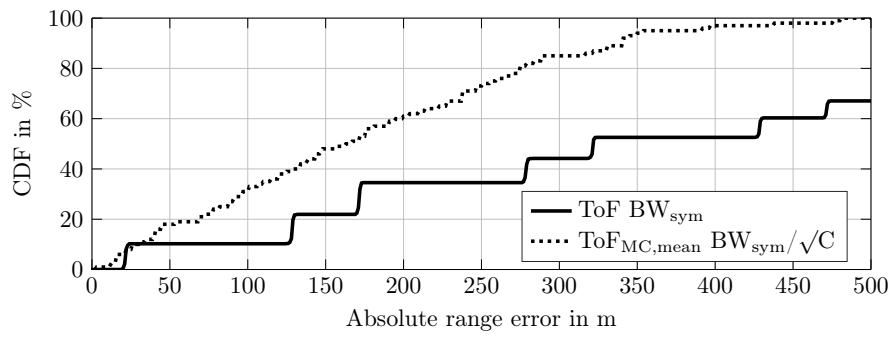

Fig. 9. Measured time-of-flight (ToF) range error and averaged time-of-flight ( ToF $_{\mathrm{MC}, \text { mean }}, C=16$ ) error at $E_{\mathrm{b}} / N_{0}=10 \mathrm{~dB}$.

performed. Delay and phase shift over $C$ channels are determined at $E_{\mathrm{b}} / N_{0}=20 \mathrm{~dB}$ and corrected on the following measurements to be range bias free.

3) Frequency flat channel: Fig. 9 shows the cumulative distribution function (CDF) for single channel time-of-flight (ToF) ranging error as well as the averaged $\mathrm{ToF}_{\mathrm{MC}}$ over $C=16$ channels. The sampling resolution due to the baseband sampling frequency is visible in form of a $150 \mathrm{~m}$ step motif in the CDF. Averaging decreases the error and smooths the CDF.

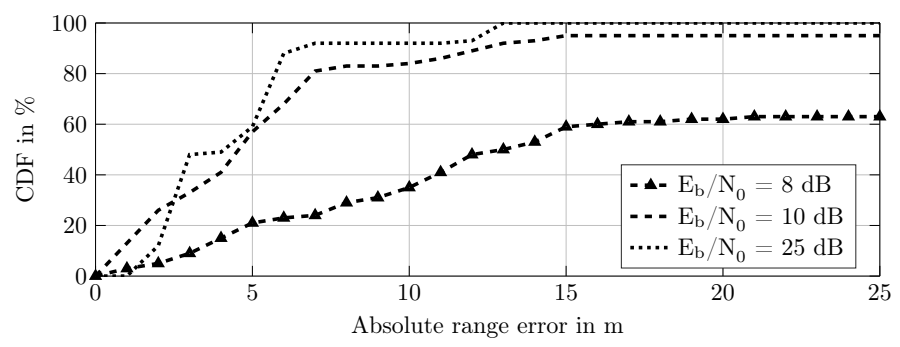

Fig. 10. Measured multi-channel phase-of-flight $(\mathrm{PoF})$ range error.

Phase-of-flight (PoF) ranging errors are, for sufficient high $E_{\mathrm{b}} / N_{0}$, more than ten times smaller than ToF ranging errors as illustrated by the CDFs in Fig. 10.

Fig. 11 compares experimental measurements to CRLBs. For low $E_{\mathrm{b}} / N_{0}$ ranging fails due to insufficient CFO estimation accuracy. For high $E_{\mathrm{b}} / N_{0}$ ToF based ranging levels out at the sampling resolution error.

Resolving the PoF range ambiguity with the $\mathrm{ToF}_{\mathrm{MC} \text {,mean }}$ measurement [14] fails in $10 \%$ of the cases due to time-offlight errors larger than the range ambiguity (compare Fig. 9). As a result, the estimated and resolved PoF range is wrong by a multiple of $R_{\max }$ for $E_{\mathrm{b}} / N_{0} \in[10 \mathrm{~dB}, 22 \mathrm{~dB}$ ], giving a large RMS error. An optimized and higher PoF range ambiguity $R_{\max }$ or adequate filtering can reduce the amount of these errors. Despite this, unresolved PoF ranging performs by a factor 10 better than $\mathrm{ToF}_{\mathrm{MC}}$, where equal symbol energy is employed. PoF ranging levels out due to non-compensated and time variant $\mathrm{CFO}$ induced errors as well as oscillator jitter.

4) Localization coverage: Results can be extrapolated to relate coverage and precision of the localization function. Defining the time-of-flight and phase-of-flight ranging accuracies $\sigma_{\mathrm{ToF}}=200 \mathrm{~m}$ and $\sigma_{\mathrm{PoF}}=10 \mathrm{~m}$ at $\left(E_{\mathrm{b}} / N_{0}\right)_{0}=10 \mathrm{~dB}$ 


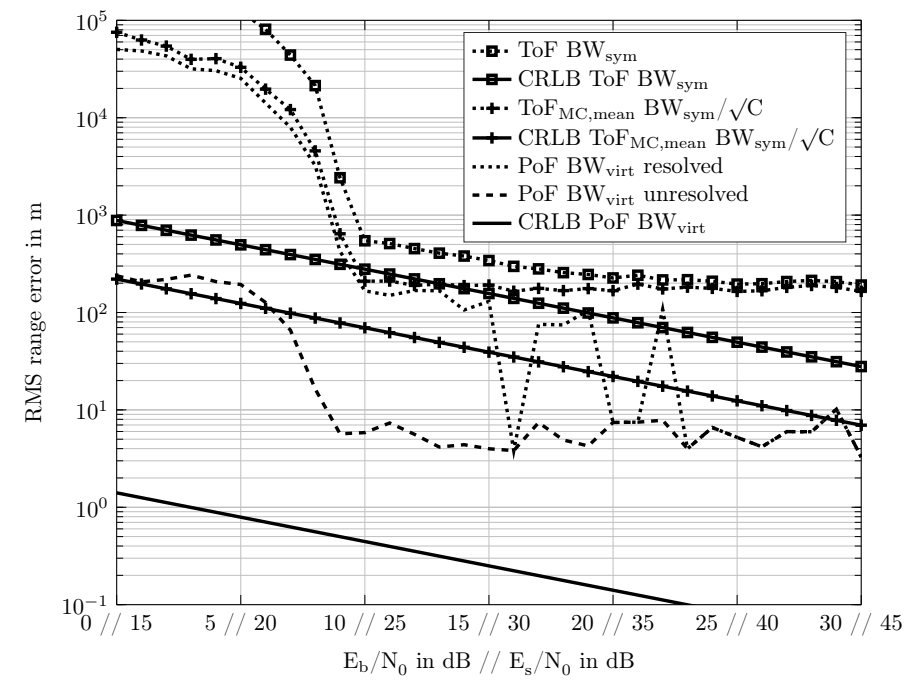

Fig. 11. Measured ranging error in a frequency flat channel with a signal bandwidth $B W_{\text {sym }}=10 \mathrm{kHz}, C=16$ channels, a virtual multi-channel bandwidth $B W_{\text {virt }}=3.0 \mathrm{MHz}$ and sampling frequency $f_{\mathrm{s}, \mathrm{BB}}=1 \mathrm{MHz}$.

as nominal ranging and also localization performances, localization coverage can be given. The receiver sensitivity is

$$
\begin{aligned}
S_{\mathrm{dBm}} & =\left(\frac{E_{\mathrm{b}}}{N_{0}}\right)_{0, \mathrm{~dB}}+(k T)_{\mathrm{dBm} / \mathrm{Hz}}+R_{\mathrm{b}, \mathrm{dBHz}}+N F_{\mathrm{dB}} \\
& =10 \mathrm{~dB}+40 \mathrm{dBHz}-174 \mathrm{dBm} / \mathrm{Hz}+2.5 \mathrm{~dB} \\
& =-121.5 \mathrm{dBm} .
\end{aligned}
$$

Link budget is $L_{\mathrm{dB}}=P_{\mathrm{TX}, \mathrm{dBm}}-S_{\mathrm{dBm}}=14 \mathrm{dBm}-$ $(-121.5 \mathrm{dBm})=135.5 \mathrm{~dB}$. According to the Hata propagation loss models [17] with $15 \mathrm{~m}$ base station and $1 \mathrm{~m}$ LPWAN radio module antenna height, a maximum coverage $R_{\text {comm }}=2.5 \mathrm{~km}$ for the suburban and $R_{\text {comm }}=7 \mathrm{~km}$ for the open rural propagation conditions can be achieved.

\section{CONCLUSION}

In this paper simulation results and preliminary experimental coherent multi-channel ranging measurements obtained with a software defined radio based ranging testbed have been presented. Precision for phase based ranging techniques improve by factor 10 to 20 compared to the time based ranging method for equal employed energy. Ranging precisions down to $10 \mathrm{~m}$ are achieved with a $10 \mathrm{kHz}$ signal and phase coherent multi-channel processing. Simulation and experimentation results agree with theoretical bounds. Algorithms perform suboptimal due to non-compensated carrier frequency offset.

Future investigation is necessary to develop adequate algorithms to compensate these errors as well as to improve range estimation in multipath scenarios. In order to understand the conditions when phase based techniques outperform classical time based approaches further simulations considering spatial filtering are required. A testbed setup with directional antennas or a multi antenna setup using the two receiver channels of the radio front end will be used to validate performances experimentally on a channel emulator and with outdoor measurements.

Complexity of multi-channel ranging can potentially be addressed by considering non-uniform channel spacing or time difference of arrival (TDoA) like approaches where the nodes become transmitter only and the base stations perform required metric estimation and range calculation.

Moreover, raw IQ data or extracted ranging metrics and the corresponding ground truth could serve as input to machine learning or deep neural network based algorithms which potentially provide, without explicit knowledge of the underlying signal model, precise ranging estimates from narrowband LPWAN radio signals.

\section{REFERENCES}

[1] U. Raza, P. Kulkarni, and M. Sooriyabandara, "Low Power Wide Area Networks: An Overview," IEEE Communications Surveys Tutorials, vol. 19, no. 2, pp. 855-873, 2017.

[2] S. M. Razavi, F. Gunnarsson, H. Ryden, A. Busin, X. Lin, X. Zhang, S. Dwivedi, I. Siomina, and R. Shreevastav, "Positioning in cellular networks: Past, present, future," in 2018 IEEE Wireless Communications and Networking Conference (WCNC), April 2018, pp. 1-6.

[3] LoRaAlliance, "Geolocation Whitepaper," 2018.

[4] Sigfox, "Sigfox to transform global asset tracking with Sigfox Geolocation, the world's cheapest Internet of Things (IoT) GPS-free geolocation service," 2017.

[5] M. Skolnik, Radar Handbook, 2nd ed. McGrawHill, 1990.

[6] Y. Karisan, D. Dardari, S. Gezici, A. A. D'Amico, and U. Mengali, "Range Estimation in Multicarrier Systems in the Presence of Interference: Performance Limits and Optimal Signal Design," IEEE Transactions on Wireless Communications, vol. 10, no. 10, pp. 33213331, October 2011.

[7] F. Wolf, C. Villien, S. de Rivaz, F. Dehmas, and J. P. Cances, "Improved multi-channel ranging precision bound for narrowband LPWAN in multipath scenarios," in 2018 IEEE Wireless Communications and Networking Conference (WCNC), April 2018, pp. 1-6.

[8] D. Vasisht, S. Kumar, and D. Katabi, "Decimeter-Level Localization with a Single WiFi Access Point," 2016.

[9] M. Pichler, S. Schwarzer, A. Stelzer, and M. Vossiek, "Multi-Channel Distance Measurement With IEEE 802.15.4 (ZigBee) Devices," IEEE Journal of Selected Topics in Signal Processing, vol. 3, no. 5, pp. 845859 , Oct 2009.

[10] A. Povalac and J. Sebesta, "Phase difference of arrival distance estimation for RFID tags in frequency domain," in 2011 IEEE International Conference on RFID-Technologies and Applications, Sept 2011, pp. 188-193.

[11] Y. Schröder, G. von Zengen, and L. Wolf, "Poster: NLOS-aware localization based on phase shift measurements," in Proceedings of the 21st Annual International Conference on Mobile Computing and Networking. ACM, 2015, pp. 224-226.

[12] S. Schwarzer, "Entwicklung eines industriellen Funkortungssystems basierend auf der kohaerenten Kombination von Kommunikationssignalen mit IEEE-802.15.4-Geraeten,” Ph.D. dissertation, Technischen Universitaet Clausthal, 2011.

[13] S. Lanzisera and K. S. J. Pister, "Burst Mode Two-Way Ranging with Cramer-Rao Bound Noise Performance," in IEEE GLOBECOM 2008 2008 IEEE Global Telecommunications Conference, Nov 2008, pp. 1-5.

[14] S. Schwarzer, M. Vossiek, M. Pichler, and A. Stelzer, "Precise distance measurement with IEEE 802.15.4 (ZigBee) devices," in 2008 IEEE Radio and Wireless Symposium, Jan 2008, pp. 779-782.

[15] U. Mengali and M. Morelli, "Data-aided frequency estimation for burst digital transmission," IEEE Transactions on Communications, vol. 45 , no. 1, pp. 23-25, Jan 1997.

[16] ETSI, Universal Mobile Telecommunications System (UMTS); Deployment aspects (3GPP TR 25.943 version 9.0.0 Release 9), European Telecommunications Standards Institute (ETSI) Std., 2010.

[17] M. Hata, "Empirical formula for propagation loss in land mobile radio services," IEEE Transactions on Vehicular Technology, vol. 29, no. 3, pp. 317-325, Aug 1980. 